University of Nebraska - Lincoln

DigitalCommons@University of Nebraska - Lincoln

August 1995

\title{
Magnetic properties of nanostructured CoSm/FeCo films
}

\author{
I.A. Al-Omari \\ University of Nebraska - Lincoln \\ David J. Sellmyer \\ University of Nebraska-Lincoln, dsellmyer@unl.edu
}

Follow this and additional works at: https://digitalcommons.unl.edu/physicssellmyer

Part of the Physics Commons

Al-Omari, I.A. and Sellmyer, David J., "Magnetic properties of nanostructured CoSm/FeCo films" (1995). David Sellmyer Publications. 98.

https://digitalcommons.unl.edu/physicssellmyer/98

This Article is brought to you for free and open access by the Research Papers in Physics and Astronomy at DigitalCommons@University of Nebraska - Lincoln. It has been accepted for inclusion in David Sellmyer Publications by an authorized administrator of DigitalCommons@University of Nebraska - Lincoln. 


\title{
Magnetic properties of nanostructured CoSm/FeCo films
}

\author{
I. A. Al-Omari and D. J. Sellmyer \\ Behlen Laboratory of Physics and Center for Materials Research and Analysis, University of Nebraska, \\ Lincoln, Nebraska 68588-0111 \\ (Received 20 January 1995)
}

\begin{abstract}
$\mathrm{Co}_{80} \mathrm{Sm}_{20} / \mathrm{Fe}_{65} \mathrm{Co}_{35}$ bilayer and multilayer films with $\mathrm{Cr}$ underlayers and overlayers have been fabricated and studied. All the samples prepared have in-plane anisotropy and the hysteresis loops were simple single loops for $t_{\mathrm{FeCo}} \leq 300 \AA$ indicating that the two phases are strongly exchange coupled. The magnetization of these samples is found to increase with increasing FeCo layer thickness for a fixed CoSm layer thickness. The coercivity, anisotropy constant, and anisotropy field for films with fixed CoSm layer thickness were found to decrease with increasing FeCo layer thickness. The magnetization squareness values of the hysteresis loops in the direction parallel to the film plane, for different CoSm and FeCo layer thicknesses, were found to be close to $S \approx 0.75$. The energy products for these samples vary from about $6 \mathrm{MGOe}$ at room temperature to $26 \mathrm{MG}$ Oe at $30 \mathrm{~K}$. The shape and the behavior of the initial loops and the minor loops suggest that wall pinning is the dominant coercivity mechanism in these films. Reversible demagnetization curves were measured and found to be consistent with the behavior expected for "exchange-spring" magnets.
\end{abstract}

\section{INTRODUCTION}

The interaction between soft and hard magnetic phases has been the subject of many recent studies. ${ }^{1-12}$ Exchange interaction between two magnetic phases, hard and soft, results in remanence enhancement and sometimes energy-product enhancement. Theoretical predictions for these enhancements have been proposed by Kneller and Hawig, ${ }^{2}$ and computer simulations and analytical calculations have been done by Skomski and Coey $^{4,5}$ and by Schrefl, Kronmüller, and Fidler. ${ }^{10}$ The origin of such enhancements is the exchange coupling between the soft and the hard phases or the so-called "exchange spring" as proposed by Kneller and Hawig. ${ }^{2}$ Several experimental studies have been published on alloyed systems such as $\mathrm{Nd}_{2} \mathrm{Fe}_{14} \mathrm{~B}$ with $\mathrm{Fe}_{3} \mathrm{~B}$ by Coehoorn De Mooij, and DeWaard, ${ }^{1}$ in which they found a maximum energy product of 12 MGOe. Withanawasam, Hadjipanayis, and $\mathrm{Krause}^{11}$ recently obtained a $(\mathrm{BH})_{\max }$ of $14 \mathrm{MG} \mathrm{Oe}$ for a fine-grained mixture of $\mathrm{Nd}_{2} \mathrm{Fe}_{14} \mathrm{~B}$ and $\alpha$-Fe prepared by the melt-spinning method.

The effects of a $\mathrm{Cr}$ underlayer and $\mathrm{Co}_{4} \mathrm{Sm}$ sputtering conditions on the structural and the magnetic properties of CoSm thin films have been studied in detail by Velu and Lambeth, ${ }^{13,14}$ Okumura et al., ${ }^{16}$ Liu and coworkers, ${ }^{17,18}$ and Sellmyer et al. ${ }^{19}$ These authors found that the $\mathrm{Cr}$ underlayer controls the magnetic properties and morphology of the CoSm layer. Shan et al. ${ }^{15}$ found that the saturation magnetization $\left(M_{s}\right)$ and the intrinsic anisotropy $\left(K_{u}\right)$ depend on the CoSm layer thickness; for example, $M_{s}$ decreases by $20 \%$ and $K_{u}$ by $67 \%$, as the layer thickness decreases from 960 to $\approx 100 \AA$. In addition, they found that by changing the sputtering conditions the coercivity mechanism can be changed from wall pinning to exchange-coupled grains. Some of the samples studied by Sellmyer et al. ${ }^{19}$ were analyzed with the mod- el of interacting particles with random anisotropy direction by Fukunaga and Inoue. ${ }^{22}$ Microstructure studies using high-resolution transmission electron microscopy (HRTEM) by Liu and co-workers ${ }^{17,18}$ showed that the $\mathrm{Cr}$ underlayer has a grain size of $250 \AA$, and the $\mathrm{Co}_{4} \mathrm{Sm}$ has a microstructure composed of crystallites of diameters 20 to $50 \AA$ distributed in an amorphous matrix. Liu and coworkers. ${ }^{17,18}$ also found that the volume fraction of the $\mathrm{Co}_{4} \mathrm{Sm}$ crystallites in the film decreases from 91 to $54 \%$ as the argon pressure during sputtering is increased from 5 to 30 mTorr.

In this paper, we report on the magnetic and structural properties of $\mathrm{Co}_{80} \mathrm{Sm}_{20} / \mathrm{Fe}_{65} \mathrm{Co}_{35}$ films with $\mathrm{Cr}$ underlayers and overlayers, where CoSm is the "hard" phase with coercivity $H_{c} \approx 2-4 \mathrm{kOe}$ (Refs. 13-15) and magnetization of $M_{s} \approx 650 \mathrm{emu} / \mathrm{cc},{ }^{13-15} \mathrm{FeCo}$ was chosen to be the "soft" phase with ( $M_{s} \approx 1934$ emu/cc) (Ref. 20) and negligible coercivity. The conditions for preparing the $\mathrm{Cr}$ underlayer were chosen to give the hard phase inplane anisotropy and the highest achievable coercivity.

\section{EXPERIMENTAL METHODS}

CoSm/FeCo bilayer and multialyers films with different $\mathrm{CoSm}$ and $\mathrm{FeCo}$ layer thicknesses, with $\mathrm{Cr}$ underlayers and coating layers, were fabricated in a multiple-gun sputtering system. All the films studied were sputtered on microscope cover-glass substrates by $\mathrm{dc}(\mathrm{CoSm}$ and $\mathrm{Cr})$ and $\mathrm{rf}(\mathrm{FeCo})$ sputtering guns. The FeCo target was made by pressing the $\mathrm{Fe}$ and $\mathrm{Co}$ powders and then sintering in vacuum $\left(\approx 10^{-6}\right.$ Torr $)$ at a temperature of $900^{\circ} \mathrm{C}$ for $\approx 1 \mathrm{~h}$. The CoSm target was made by pressing CoSm particles and Co powder together and then sintering in vacuum $\left(\approx 10^{-6}\right.$ Torr $)$ at a temperature of $1100^{\circ} \mathrm{C}$ for $\approx 0.5 \mathrm{~h}$. The $\mathrm{Cr}$ target was commercially obtained and had $99.9 \%$ purity. The base pres- 
sure of the sputtering system was $2-3 \times 10^{-7}$ Torr and the Ar pressure during sputtering was optimized to give the highest coercivity; it varied from $12 \mathrm{mTorr}$ for the $\mathrm{Cr}$ underlayer to $30 \mathrm{mTorr}$ for $\mathrm{CoSm}$ and FeCo. The sputtering rates and powers were $\approx 5.4 \AA / \mathrm{sec}$ and $100 \mathrm{~W}$ for $\mathrm{Cr}, \approx 1.4 \AA / \mathrm{sec}$ and $15 \mathrm{~W}$ for $\mathrm{CoSm}$ and $\approx 1.9 \AA / \mathrm{sec}$ and $50 \mathrm{~W}$ for $\mathrm{FeCo}$. In each vacuum run 12 samples were made.

X-ray diffraction was used to study the structural properties of the films. The magnetic properties were studied over the temperature range of $30-300 \mathrm{~K}$ with an alternating gradient force magnetometer with a maximum field of $10 \mathrm{kOe}$.

\section{RESULTS AND DISCUSSION}

\section{A. Structural properties}

$\mathrm{X}$-ray-diffraction measurements on the $\mathrm{Cr}$ underlayer sputtered onto a glass substrate at $12 \mathrm{mTorr}$ Ar pressure showed only the (110) peaks of the bcc structure. This underlayer structure tends to control the magnetic properties of the CoSm layer by giving large coercivity and in-plane random anisotropy. X-ray diffraction for the CoSm layer showed no diffraction peaks. This is consistent with other observations by Shan et al. ${ }^{15}$ since the CoSm crystallites are too small $(20-50 \AA)$ to show diffraction peaks. FeCo has also been measured by x-ray diffraction and the diffraction patterns for this layer on glass substrate or on ( $\mathrm{Cr}$ on glass substrate) showed the bcc structure. HRTEM studies by Liu and coworkers $^{17,18}$ showed that the CoSm has crystallites with dimensions in the range of 20-50 $\AA$ embedded in an amorphous matrix of CoSm alloy. They found also that the CoSm nanocrystallites have a disordered close-packed hexagonal structure with varying stacking sequences. The hexagonal axes of the crystallites (the easy anisotropy axes) are found to be randomly oriented in the plane of the film.

\section{B. Magnetic properties}

\section{Bilayers of different FeCo layer thicknesses}

Samples of the form glass: $\operatorname{Cr}(928 \AA): \mathrm{Co}_{80} \mathrm{Sm}_{20}(70$ $\AA) / \mathrm{Fe}_{65} \mathrm{Co}_{35}(x \AA): \operatorname{Cr}(111 \AA)$ with $x$ between 0 and 250 $\AA$ have been made and studied. Figure 1 shows typical magnetization loops for one of the samples studied. From Fig. 1(a) we see that the films have in-plane anisotropy. Figures $1(\mathrm{~b})$ and 1 (c) show the initial curve, hysteresis loop, and minor loops with an expanded $H$ axis. Figure 1(c) displays features suggestive of either wall pinning ${ }^{15,19,21}$ or a Stoner-Wohlfarth-like particle magnetization reversal mechanism. From the initial loops it seems likely that wall pinning is the dominant mechanism which is the same mechanism for CoSm films prepared under the same mechanism for CoSm films prepared under the same conditions by Shan et al. ${ }^{15}$ By plotting the dependence of the coercivity on the maximum applied field $\left(H_{\max }\right)$ from Fig. 1(c) we found that there is a small change in $H_{c}$ for a maximum fields less than the pinning
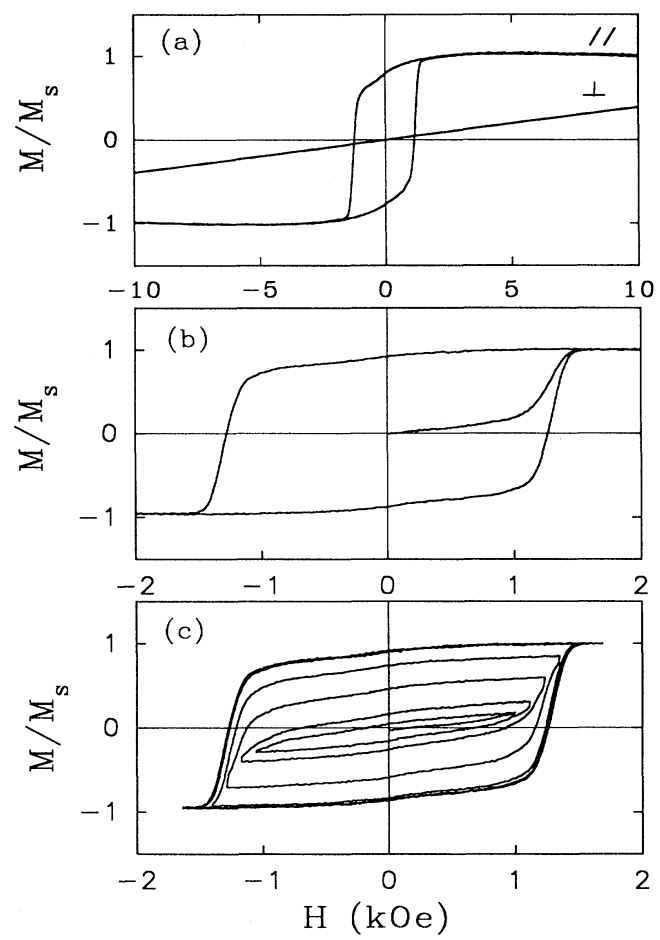

FIG. 1. Typical magnetization curves for $\operatorname{Cr}(928 \AA)$ : $\mathrm{Co}_{80} \mathrm{Sm}_{20}\left(70 \AA / \mathrm{Fe}_{65} \mathrm{Co}_{35}(31.1 \AA): \mathrm{Cr}(111 \AA\right.$ ) (a) parallel and perpendicular loops (b) initial loop and hysteresis loop (c) minor hysteresis loops. Note the expanded $x$-axis scale for (b) and (c).

field $\left(H_{p}\right)$ and the change becomes rapid when $H_{\max }$ is equal to $H_{p}$. This result indicated that wall pinning is the dominant coercivity mechanism in these films as pointed out by McCurrie. ${ }^{30}$ The coercivity and the saturation magnetization with the applied field parallel (\|) to the film plane as a function of FeCo thickness are shown in Fig. 2. The coercivity and magnetization values for $x=0$ are in good agreement with other values of Velu

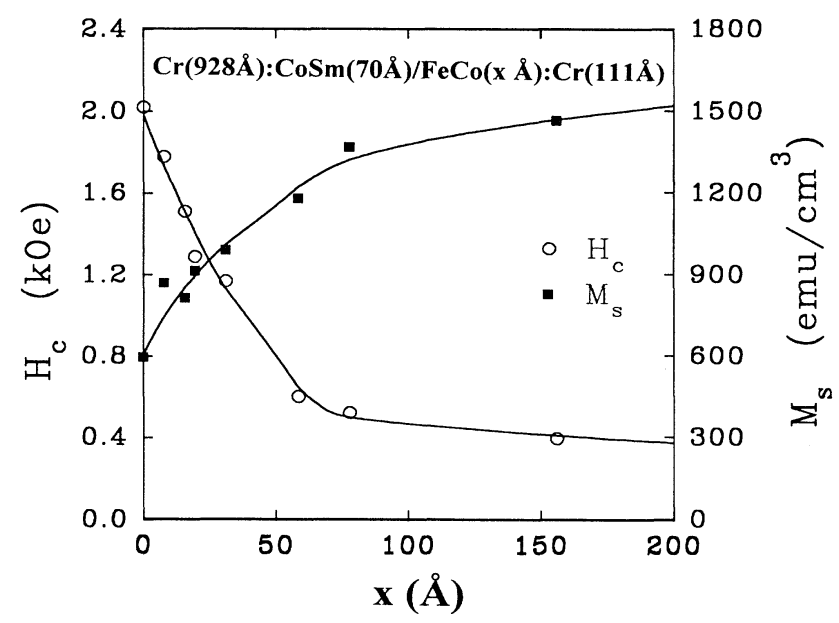

FIG. 2. Dependence of the magnetization and the coercivity on the $\mathrm{Fe}_{65} \mathrm{Co}_{35}$ layer thickness. The solid lines are drawn as a guide to the eye. 
and Lambeth ${ }^{13}$ and Shan et al. ${ }^{15}$ The saturation magnetization for $\mathrm{FeCo}$ was found to be $\approx 1850 \mathrm{emu} / \mathrm{cc}$, which is similar to the value of $1934 \mathrm{emu} / \mathrm{cc}$ by Weiss and Forrer. ${ }^{20}$ As the thickness $(x)$ of the soft phase (FeCo) is increased, the coercivity goes down and the magnetization goes up; these changes are rapid up to $x=65 \AA$, where the two phases become approximately equal in thickness. Above $x \approx 70 \AA$ the changes in $M_{s}$ and $H_{c}$ are slower. The magnetization is fitted with the formula

$$
\overline{\boldsymbol{M}}_{S}=f_{s} \boldsymbol{M}_{s}+f_{h} \boldsymbol{M}_{h},
$$

which is appropriate for a superposition of the two phases. Here $f_{s}, M_{s}, f_{h}$, and $M_{h}$ are the percentages and the magnetizations of the soft and the hard phases, respectively. Figure 3 shows the fitted and the experimental data, and there is reasonable agreement between the experimental data and Eq. (1). All of the films reported in this paper showed simple loops indicative of strong exchange coupling between the two phases. For $t_{\mathrm{FeCo}} \geq 300$ $\AA$, the loops had the character of two independently switching phases.

Skomski and Coey $^{5}$ have predicted that the nucleation field in an exchange-spring magnet is given by

$$
H_{N}=\left(2 \bar{K}_{1} / \bar{M}_{S}\right)-D \bar{M}_{S},
$$

where $\bar{M}_{S}$ is given by Eq. (1), $\bar{K}_{1}$ is the composite or net anisotropy constant for the material ( $\approx K_{u}$ in our case), and $D$ is the microscopic demagnetization factor. Equation (2) might be generalized following Kronmüller, Durst, and Sagawa ${ }^{23}$ to the form

$$
H_{c}(x)=\alpha(x)\left(2 \bar{K}_{1} / \bar{M}_{S}\right)-D_{\mathrm{eff}}(x) \bar{M}_{S},
$$

where $\alpha(x)$ is a parameter depending on reversal mechanism, misorientation of grains, etc., $D_{\text {eff }}(x)$ represents an effective demagnetization coefficient relevant for the particular nanostructure, and $x$ is the thickness of the soft phase. In a typical analysis of the type used by Kronmüller, Durst, and Sagawa, ${ }^{23}$ a plot of $H_{c} / M_{s}$ versus $2 K_{1} / M_{s}^{2}$ is made to study the reversal mechanism

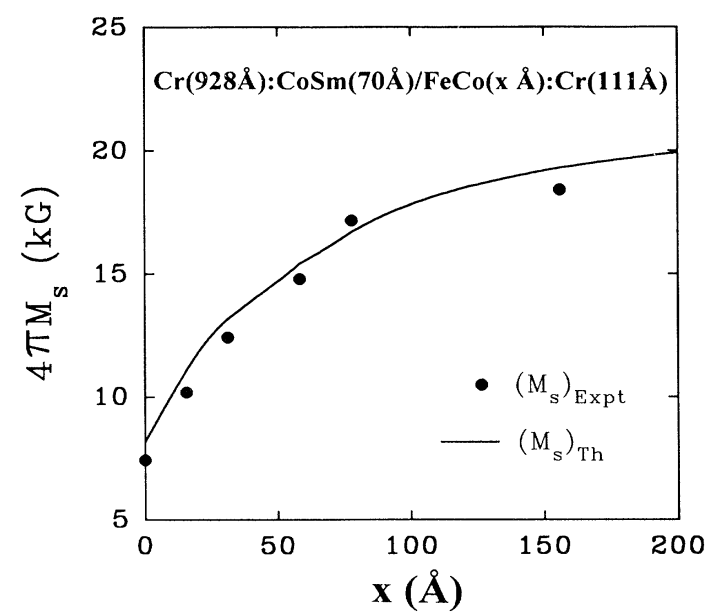

FIG. 3. Dependence of the magnetization on the $\mathrm{Fe}_{65} \mathrm{Co}_{35}$ layer thickness. The solid line represent the fitting. through the parameters $\alpha$ and $D_{\text {eff }}$.

For each sample denoted by the thickness $(x), H_{c}$ depends on the temperature. Our results as well as those of Shan et al. ${ }^{15}$ indicate that wall pinning is the dominant coercivity mechanism in our samples, so Eq. (3) can be written as

$$
H_{c}=\beta\left(2 K_{1}^{1.5} / M_{s}\right)-D_{\mathrm{eff}} M_{S} ;
$$

this form of Eq. (3) is appropriate for wall pinning when the size of the pinning site $\left(r_{0}\right)$ is small compared to the domain-wall thickness $(\delta)$. Analysis by Shan et al. ${ }^{15}$ showed that for a CoSm sample with no soft phase present $r_{0} \approx 5 \AA$, which is much less than $\delta \approx 150 \AA$. Figure 4 shows $\left(H_{c} / \bar{M}_{S}\right)$ versus $\left(2 \bar{K}_{1}{ }^{1.5} / \bar{M}_{S}{ }^{2}\right)$ for a sample with $x=31.1 \AA$. The solid line is a linear fit to the data, and the intercept of this line gives the demagnetization factor $\left(D_{\text {eff }}\right)$, while the slope gives the microstructural reduction parameter $(\beta)$ according to the expression given by Kronmüller, Durst, and Sagawa. ${ }^{23}$ From the fitting we found that $D_{\text {eff }}=-4.0,-0.31$, and $\beta=0.55$ $\times 10^{-5}(\mathrm{erg} / \mathrm{cc})^{1 / 2}, 3.58 \times 10^{-5}(\mathrm{erg} / \mathrm{cc})^{1 / 2}$ for $x=0$ and $31.1 \AA$, respectively. The different values of $D_{\text {eff }}$ and $\beta$ for different values of $x$ are due to differences in the nanostructure because of the difference in the thickness of the soft phase. The negative values of $D_{\text {eff }}$ are due to the ferromagnetic exchange interactions between the particles in these films, which is confirmed by other means as we shall discuss subsequently.

We now assume that the factor $(D)$ in Eq. (2) is equal to $D_{\text {eff }}$, found from the temperature-dependent analysis of Eq. (3), in order to compare the experimental $H_{c}$ values with those predicted by Eq. (2) at room temperature for a composite system. We calculated the ratios of the experimental coercivity values to the calculated nucleation values given by Eq. (2) for the two samples. The results are $\left[H_{c}(0 \AA) / H_{n}(0 \AA)\right]=0.11$ and $\left[H_{c}(31.1 \AA) /\right.$ $\left.H_{n}(31.1 \AA)\right]=0.12$. From these results we see that these ratios for different thicknesses of the soft phase are equal within the experimental errors. This indicates that there is a reduction in the theoretical coercivities, predicted by

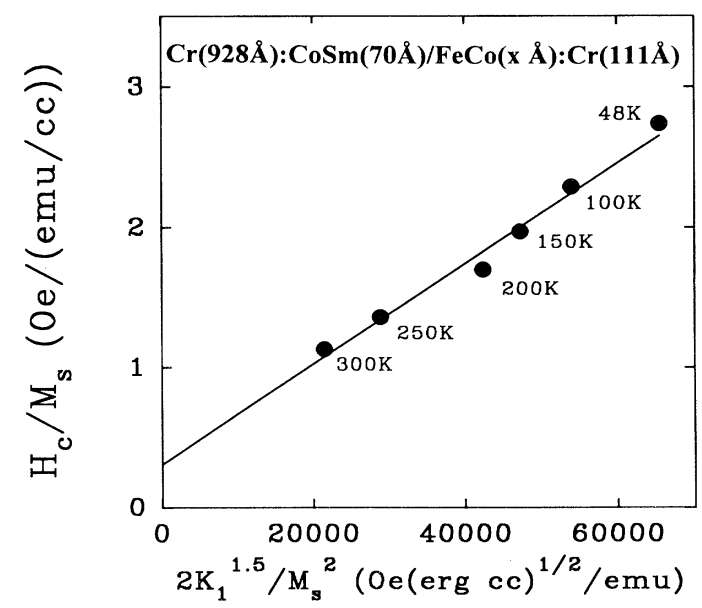

FIG. 4. Dependence of $H_{c} / M_{s}$ on $2 K_{1}^{1.5} / M_{s}^{2}$. The solid lines represent the fitting. 
Eq. (2) for a composite system, by a factor of about 0.1 . This is not surprising in comparison with observations by Kronmüller, Durst, and Sagawa ${ }^{23}$ and others for other permanent magnets when pinning is the dominant coercivity mechanism, and where the reduction factor typically is between zero and 0.3. A possible reason for the reduction factor with and without the soft phase is the misorientation of the easy axes of the grains, in which the moments of the hard phase are randomly oriented in the plane of the film. It must be emphasized that the above analysis is very rudimentary because of our inability to understand fundamentally the two parameters $\alpha$ and $D_{\text {eff }}$ in Eq. (3).

The measured anisotropy fields $\left(H_{A}\right)$ for different FeCo layer thicknesses are estimated from the extrapolated intersection of the perpendicular and parallel loops. $H_{A}$ decreases rapidly by $25 \%$ as $x$ increases from 0 to 50 $\AA$ and then the change becomes slow for $x>50 \AA$, where the thickness of the soft phase becomes comparable with or larger than that of the hard phase. The same behavior also was observed for the intrinsic anisotropy $\left(K_{u}\right)$ as shown in Fig. 5. The rapid decrease in $H_{A}$ and $K_{u}$ when $x<50 \AA$ and the slow decrease for $x>50 \AA$ can be understood in terms of the initial rapid changes in $M_{s}$ and $H_{c}$ for $x<50 \AA$ and the subsequent slow changes in $M_{s}$ and $H_{c}$ for $x>50 \AA$, as seen in Fig. 2. The maximum energy product $(B H)_{\max }$ was calculated for these samples from the second quadrant of the hysteresis loops. The results are shown in Fig. 6. We see that there is an enhancement in $(B H)_{\max }$ when $x \leq 50 \AA$; then it decreases as $x$ increases. The enhancement in $(B H)_{\max }$ is due to the enhancement in $M_{s}$ as seen in Fig. 2. The decrease in $(B H)_{\max }$ for $x>50 \AA$ is due largely to the decrease in the coercivity. Theoretical calculations by Skomski and Coey ${ }^{4-6}$ and computer simulations by Schrefl, Kronmüller, and Fidler ${ }^{10}$ predict that there can be an increase in $(B H)_{\max }$ when hard and soft phases are exchange coupled. In these theoretical calculations they assume ideal conditions where the hard phase is crystal-

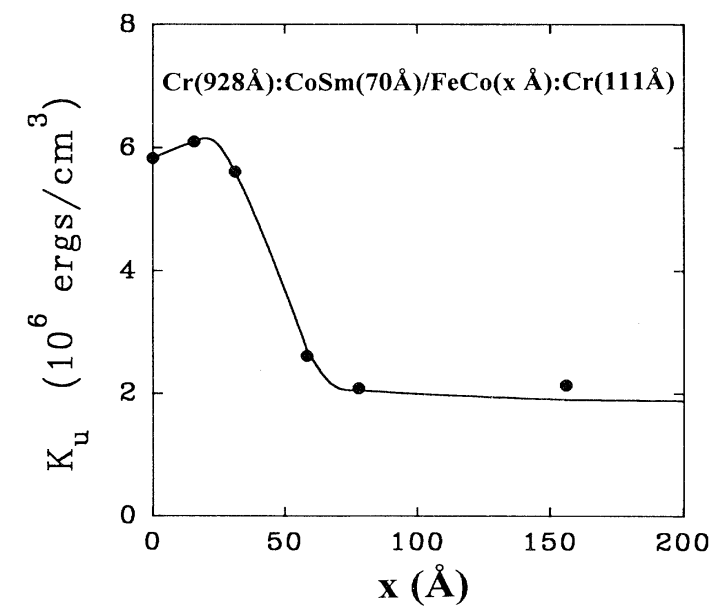

FIG. 5. Dependence of the anisotropy constant on the $\mathrm{Fe}_{65} \mathrm{Co}_{35}$ layer thickness. The solid line is drawn as a guide to the eye.

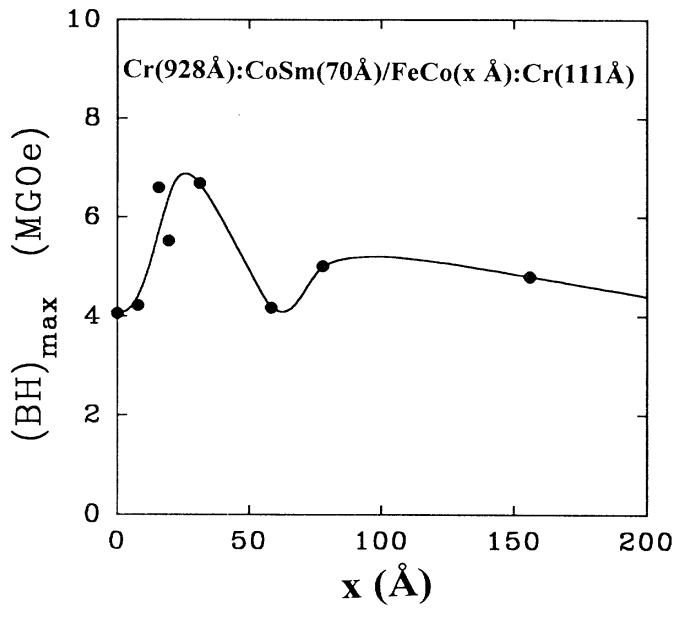

FIG. 6. Dependence of the maximum energy product on the $\mathrm{Fe}_{65} \mathrm{Co}_{35}$ layer thickness. The solid line is drawn as a guide to the eye.

line, the easy axes of the hard phase is aligned, the size of the soft grains less than twice the domain-wall thickness of the hard phase $\left[\delta=\pi\left(A / K_{1}\right)^{1 / 2}\right]$, where $A$ is the exchange parameter, and the soft and hard phases have sharp boundaries. In our samples about $65 \%$ of the volume of the hard phase is nanocrystalline particles embedded in an amorphous matrix.

The domain-wall thickness for our CoSm films is of the order of (120-180) $\AA$ and it depends on the thickness of the film because $K_{1}$ increases with thickness. ${ }^{15}$ Results for samples with CoSm thickness $186 \AA$ and different FeCo thickness showed that there is an increase of $22 \%$ in $(B H)_{\max }$ when $t_{\mathrm{FeCo}} \leq 300 \AA$ and a decrease, relative to $\mathrm{CoSm}$, when $t_{\mathrm{FeCo}} \geq 300 \AA$. This thickness of the soft phase $(300 \AA)$ is approximately twice the domain-wall thickness of the hard phase $(2 \delta)$; so the decrease in $(B H)_{\max }$ can be perhaps attributed to the decoupling be-

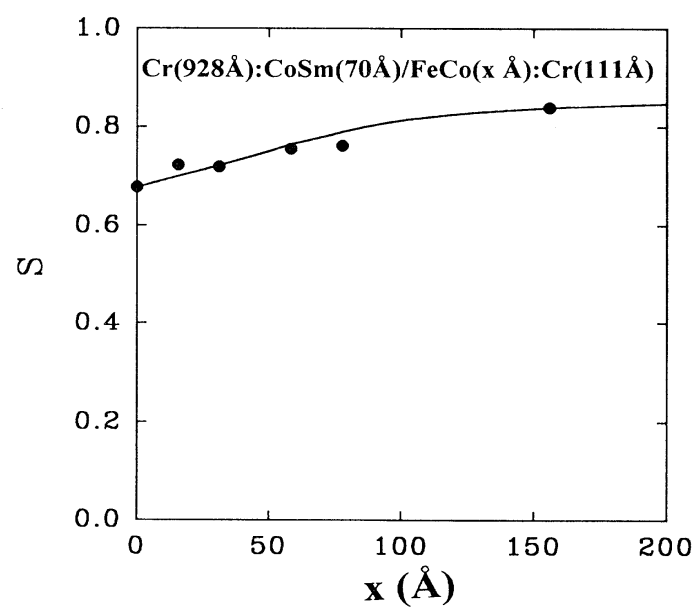

FIG. 7. Dependence of the magnetization squareness on the $\mathrm{Fe}_{65} \mathrm{Co}_{35}$ layer thickness. The solid lines are drawn as a guide to the eye. 
tween the two phases when $t_{\text {soft phase }} \geq 2 \delta_{\text {hard phase, which }}$ is consistent with the theoretical results of Skomski and Coey $^{4,5}$ and Schrefl, Kronmüller, and Fidler. ${ }^{10}$

Figure 7 shows the dependence of the magnetization squareness ( $S \equiv M_{r} / M_{s}$ where $M_{r}$ is the remanence) on FeCo thickness $x$. The change in $S$ is very small and the average value is about 0.75 . This value for $S$ is characteristic of exchange-spring magnets where $S$ should be more than $0.5^{(2)}$. The dc magnetization curves were measured by first saturating the sample in a high field, applying a negative field, and then reducing the field to zero and measuring the remanence $\left[M_{r}(H)\right]$. From these measurements we determined $\Delta M(H)$ which is equal to $\left[M_{r}(H)-M_{r}(0)\right]$, where $H$ is the reverse field. A plot of $-[\Delta M(H)] / 2 M_{r}$ versus the reverse field is shown in Fig. 8. From this figure we see that the change in $\Delta M(H)$ is small for small fields, then after a certain field it becomes rapid. The small change corresponds to the reversible region of the demagnetization curve and the rapid change is due to the irreversible rotation of the magnetic moments. This is a typical behavior for exchange-spring magnets and it has been observed by Kneller and Hawig, ${ }^{2}$ and by Withanaswasam, Hadjipanayis, and Krause. ${ }^{11}$

The isothermal remanence $M_{r}(H)$ and the demagnetization remanence $M_{d}(H)$ were measured to study the magnetic interactions; $M_{r}(H)$ is measured by a progressive magnetization of an initially ac-demagnetized sample and $M_{d}(H)$ is measured by a progressive demagnetization from a previously saturated state. ${ }^{25-29}$ By using the technique of Henkel plots ${ }^{24}\left(M_{d}\right.$ versus $M_{r}$ ), we can study the magnetic interactions. This technique was developed by Kelly et al., ${ }^{25}$ where by plotting the interaction-based deviation parameter $\Delta I(H)$ versus the applied field, the character of the interactions can be investigated. The relation between $\Delta I(H)$ and $I_{d}$ and $I_{r}$ is given by the following equation: ${ }^{25-29}$

$$
\Delta I(H)=I_{d}(H)-\left[1-2 I_{r}(H)\right],
$$

where $I_{d}$ and $I_{r}$ are the magnetization values $\left(M_{d}\right.$ and

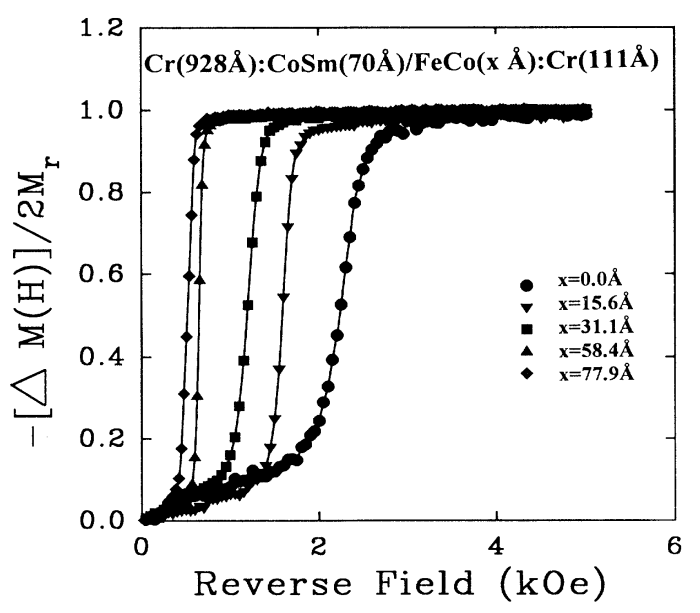

FIG. 8. Dependence of $-[\Delta M(H)] / 2 M_{r}$ on the reverse field for different $x$ values.

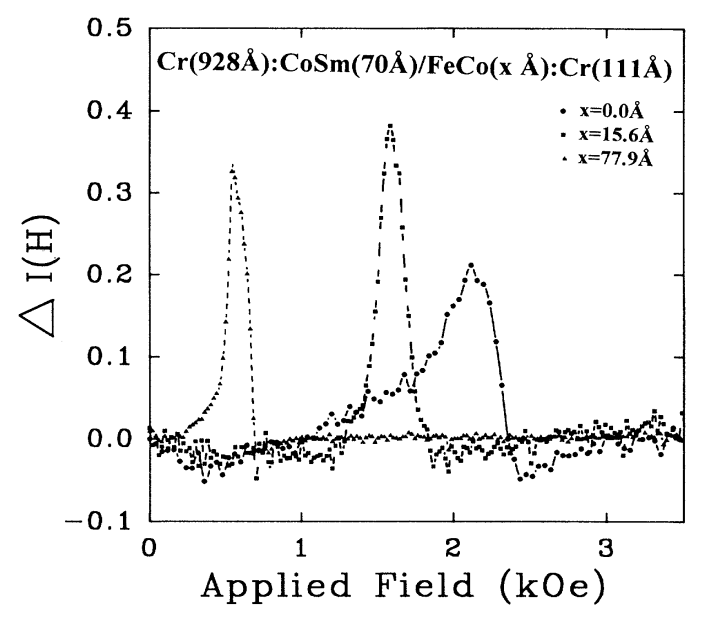

FIG. 9. $\Delta I(H)$ curves for different values of $x$.

$M_{r}$ ) normalized to the saturation values. Figure 9 shows the dependence of $\Delta I$ on $H$ for different values of $x$. It is seen that $\Delta I$ has positive values, which indicate a ferromagnetic interaction, or positive exchange coupling, in these films. ${ }^{25-29}$ This is consistent with our previous discussion. For $x=0$ the peak is broad, and the width becomes narrower as $x$ increases; this is due to the increase in the squareness $(S)$ of the films, which is consistent with other observations by Kelly et al. ${ }^{25}$ for Co-P thin films. The change in the position of the peak is due to the change in the coercivity of the films.

The coercivity versus the temperature was studied to investigate the coercivity mechanism in these films. Figure 10 shows the temperature dependence of the coercivity, which shows that $H_{c}$ increases with decreasing temperature. The coercivities of samples with $x>50 \AA$ were found to have a weaker temperature dependence than those with $x=15.6$ and $31.1 \AA$, because of the large

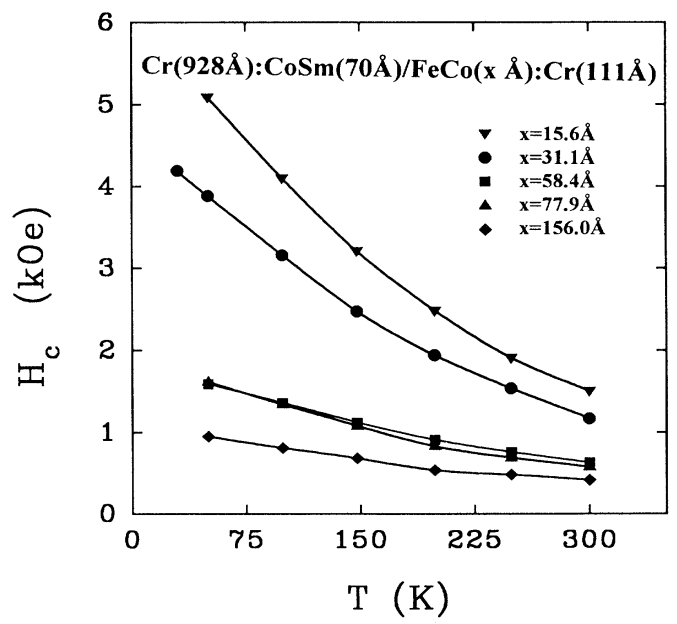

FIG. 10. Dependence of the coercivity on the temperature for different $x$ values. The solid lines are drawn as a guide to the eye. 


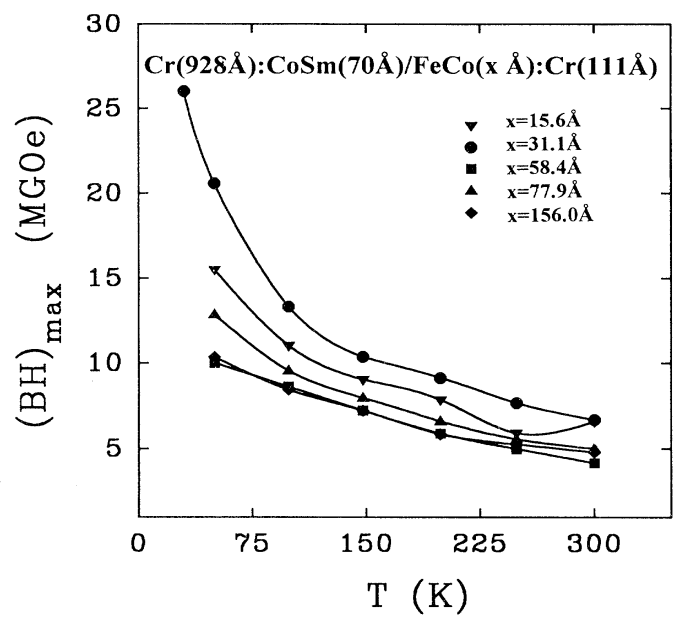

FIG. 11. Dependence of the maximum energy product on the temperature for different $x$ values. The solid lines are drawn as a guide to the eye.

amount of the soft phase in the former samples. Such behavior, and the behaviors in Fig. 1 and Fig. 4, are typical behaviors of wall pinning which is the dominant coercivity mechanism in CoSm films as shown by Shan et al. ${ }^{15}$ The magnetization values of these films for temperatures down to $30 \mathrm{~K}$ were found to have small increase relative to the room-temperature values. The temperature dependence of the maximum energy product also was determined with the results shown in Fig. 11. The maximum energy products for samples with those of small $x$ showed a stronger temperature dependence than those with large $x$. For example, $(B H)_{\max }$ for $x=31.1 \AA$ increases from 6.7 MG Oe at $T=300 \mathrm{~K}$ to $26.0 \mathrm{MG}$ Oe at $T=30 \mathrm{~K}$. Samples with CoSm layer thicknesses of 240 , $500,750,1000$, and $1500 \AA$ with different layer thicknesses of FeCo showed the same physical behaviors as $\mathrm{CoSm}(70 \AA)$ with different FeCo layer thicknesses; $H_{c}$

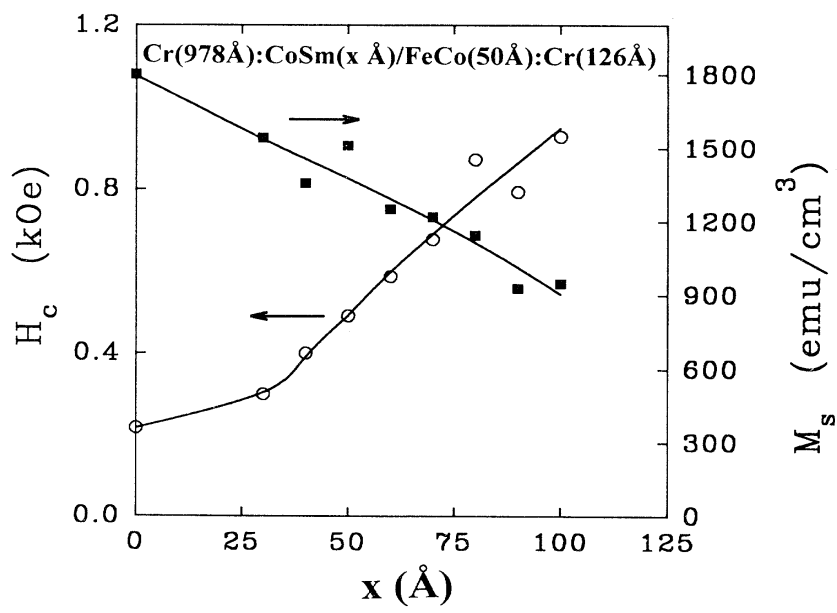

FIG. 12. $\mathrm{Co}_{4} \mathrm{Sm}$ layer thickness dependence of magnetization and coercivity. The solid lines are drawn as a guide to the eye.

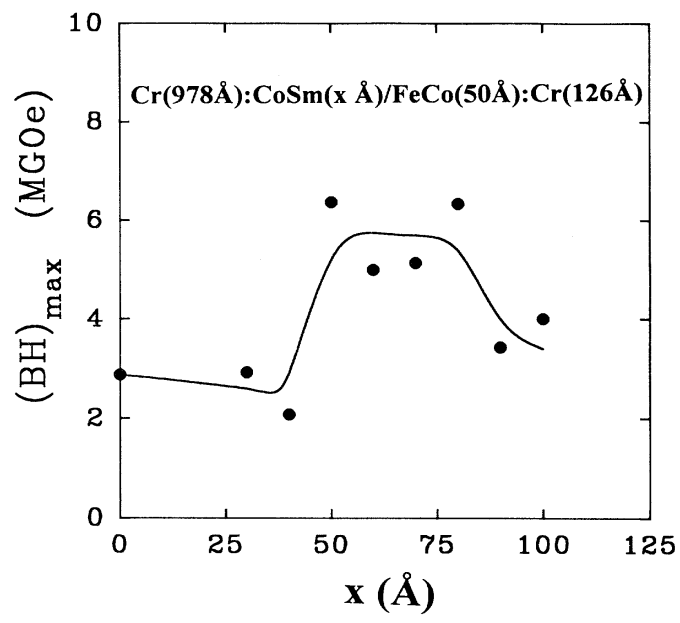

FIG. 13. Dependence of the maximum energy product on the $\mathrm{SmCo}_{4}$ layer thickness. The solid line is drawn as a guide to the eye.

decreases and $M_{s}$ increases by increasing the $\mathrm{FeCo}$ layer thickness.

\section{Bilayers with different CoSm layer thicknesses}

Samples of the form glass: $\operatorname{Cr}(978 \AA): \operatorname{CoSm}(x$ $\AA) / \mathrm{FeCo}(50 \AA)$ : $\mathrm{Cr}(126 \AA$ ) with $x$ between 0 and $100 \AA$ were prepared and studied. Figure 12 shows $M_{s}$ and $H_{c}$ for films with different $x$. $M_{s}$ decreases and $H_{c}$ increases with increasing CoSm layer thickness which is a typical behavior for such combinations. The hysteresis loops for all the samples studied were simple single loops with no evidence for two $H_{c}$ values, so that the two phases have strong exchange coupling. $S$ was also of the order of 0.75 for these samples. Figure 13 shows the dependence of $(B H)_{\max }$ on $x$. There is an enhancement in $(B H)_{\max }$ when $x \approx 50 \mathrm{~A}$ which is equal to the thickness of the soft phase. This enhancement is due to the enhancement in $H_{c}$ with increasing $x$, where we have a rapid increase in $H_{c}$ for 30 $\AA \leq x \leq 80 \AA$ as seen in Fig. 12.

\section{Multilayers of $\mathrm{Co}_{80} \mathrm{Sm}_{20}$ and $\mathrm{Fe}_{65} \mathrm{Co}_{35}$ films}

Samples of the form glass: $\operatorname{Cr}(928 \AA)$ : $[\operatorname{CoSm}(x \AA)$ $/ \operatorname{FeCo}(y \AA)]_{n}: \operatorname{Cr}(111 \AA)$ with different values for $x, y$, and $n$ were made and studied; some of these are shown in Table I. As we see from Table I, $H_{c}$ for multilayer sam-

TABLE I. Magnetization $\left(M_{s}\right)$ and coercivity $\left(H_{c}\right)$ of $\mathrm{Cr}(928$ $\AA$ ): $\left.\left[\mathrm{Co}_{80} \mathrm{Sm}_{20}(x \AA) / \mathrm{Fe}_{65} \mathrm{Co}_{35}(y \AA)\right]_{n}: \operatorname{Cr}(111) \AA\right)$.

\begin{tabular}{rrrcc}
\hline \hline$x(\AA)$ & $y(\AA)$ & $n$ & $M_{s}(\mathrm{emu} / \mathrm{cc})$ & $H_{c}(\mathrm{kOe})$ \\
\hline 240 & 0 & 0 & 600 & 2.43 \\
45 & 45 & 34 & 1365 & 0.37 \\
150 & 150 & 10 & 1256 & 0.43 \\
88 & 74 & 30 & 1490 & 0.38 \\
139 & 116 & 14 & 1463 & 0.53 \\
64 & 31 & 5 & 1195 & 0.51 \\
25 & 90 & 27 & 1857 & 0.06 \\
\hline \hline
\end{tabular}


ples with approximately equal layer thickness, for the hard and the soft phase, is about $0.4 \mathrm{kOe}$ and $M_{s} \approx 1400$ $\mathrm{emu} / \mathrm{cc}$ which is in agreement with the superposition principle as mentioned above. Samples with $y \gg x$ showed small coercivities $(<100 \mathrm{Oe})$, while samples with $x>y$ were found to have larger coercivities ( $>1000 \mathrm{Oe}$ ).

\section{CONCLUSIONS}

Samples of the form glass: $\mathrm{Cr}(\approx 950 \AA):\left[\mathrm{Co}_{80} \operatorname{Sm}_{20}(x\right.$ $\left.\AA) / \mathrm{Fe}_{65} \mathrm{Co}_{35}(y \AA)\right]_{n}: \mathrm{Cr}(\approx 115 \AA)$ with different layers thickness ( $x$ and $y$ ) and different bilayer number $(n)$ were prepared and studied. All samples were found to have in-plane anisotropy. When $y$ is increased keeping $x$ constant it is found that there is an enhancement in $M_{s}$ and hence $(B H)_{\max }$ up to a certain value of $y$. Increasing $x$ while keeping $y$ fixed results in an enhancement in $H_{c}$ and some enhacement in $(B H)_{\max }$. The anisotropy field, anisotropy constant and coercivity for samples with fixed $x$ were found to decrease with increasing $y$. All the samples studied with $y \leq 300 \AA$ were found to have simple single loops with $S \geq 0.70$ which indicates that there is a strong exchange coupling between the hard and the soft phases.
The demagnetization curves generally are reversible, which is a characteristic of exchange-spring magnets. Limited contact was made with theoretical expressions for magnetization and coercivity for exchange-spring magnets, but the lack of perfect easy-axis alignment and nanocomposite character of our CoSm films made detailed comparison difficult. Such samples with relatively low rare-earth concentration $(\approx 12 \%)$ and in-plane anisotropy might be used in devices that require relatively small $(B H)_{\max }$. Also they might have potential for highdensity magnetic recording media. By choosing the thickness of the soft phase to be relatively small compared to the thickness of the hard phase it may be possible to obtain the high coercivity $(2.5-4.5 \mathrm{kOe})$ and small grain size required for such applications.

\section{ACKNOWLEDGMENTS}

We are grateful to Professor S. S. Jaswal, Professor R. D. Kirby, Dr. Z. S. Shan, and S. S. Malhotra for helpful assistance and discussions. We thank the United States Department of Energy for support under Grant No. DE-FG2-86ER45262.
${ }^{1}$ R. Coehoorn, D. B. De Mooij, and D. De Waard, J. Magn. Magn. Mater. 80, 101 (1989).

${ }^{2}$ Ekart F. Kneller and Reinhard Hawig, IEEE Trans. Magn. 27, 3588 (1991).

${ }^{3}$ K. O'Donnell, C. Kuhrt, and J. M. D. Coey, J. Appl. Phys. 76, 7068 (1994).

${ }^{4}$ Ralph Skomski and J. M. D. Coey, Phys. Rev. B 48, 15812 (1993).

${ }^{5}$ R. Skomski and J. M. D. Coey, IEEE Trans. Magn. 29, 2860 (1993).

${ }^{6}$ R. Skomski, J. Appl. Phys. 76, 7059 (1994).

${ }^{7}$ J. M. Yuo, T. S. Chin, and S. K. Chen, J. Appl. Phys. 76, 7071 (1994).

${ }^{8}$ K. Ounadjela and G. Suran, J. Appl. Phys. 67, 3244 (1988).

${ }^{9}$ T. Schrefl, H. F. Schmidts, J. Fidler, and H. Kronmüller, IEEE Trans. Magn. 29, 2878 (1993).

${ }^{10}$ T. Schrefl, H. Kronmüller, and J. Fidler, J. Magn. Magn. Mater. 127, L273 (1993).

${ }^{11}$ L. Whitanawasam, G. C. Hadjipanayis, and R. F. Krause, J. Appl. Phys. 75, 6646 (1994).

${ }^{12}$ S. Hirosawa, H. Kanekiyo, and M. Uehara, J. Appl. Phys. 73, 6488 (1993).

${ }^{13}$ E. M. T. Velu and D. N. Lambeth, J. Appl. Phys. 69, 5175 (1991)

${ }^{14}$ E. M. T. Velu and D. N. Lambeth, IEEE Trans. Magn. 28, 3249 (1992).

${ }^{15}$ Z. S. Shan et al. (unpublished).

${ }^{16}$ Y. Okumura, H. Fujimori, O. Suzuki, N. Hosoya, X. Yang, and H. Morita, IEEE Trans. Magn. 30, 4038 (1994).
${ }^{17}$ Y. Liu, B. Robertson, Z. S. Shan, S. S. Malhotra, M. J. Yu, S. K. Renukunta, S. H. Liou, and D. J. Sellmyer, IEEE Trans. Magn. 30, 4035 (1994).

${ }^{18}$ Y. Liu, B. Robertson, Z. S. Shan, S. H. Liou, and D. J. Sellmeyer, J. Appl. Phys. 77, 3831 (1995).

${ }^{19}$ D. J. Sellmyer, Z. S. Shan, Y. Liu, S. H. Liou, S. S. Malhotra, and B. W. Robertson (unpublished).

${ }^{20}$ P. Weiss and R. Forrer, Ann. Phys. (Paris) 12, 279 (1929).

${ }^{21}$ Joseph J. Becker, IEEE Trans. Magn. MAG-12, 965 (1976).

${ }^{22}$ Hirotoshi Fukunaga and Hiroshi Inoue, Jpn. J. Appl. Phys. 31, 1347 (1992).

${ }^{23}$ H. Kronmüller, K. D. Durst, and M. Sagawa, J. Magn. Magn. Mater. 74, 291 (1988).

${ }^{24}$ O. Henkel, Phys. Status Solidi 7, 919 (1964).

${ }^{25}$ P. E. Kelly, K. O'Grady, P. I. Mayo, and R. W. Chantrell, IEEE Trans. Magn. 25, 3881 (1989).

${ }^{26}$ K. O'Grady, R. W. Chantrell, and I. L. Sanders, IEEE Trans. Magn. 29, 286 (1993).

${ }^{27}$ P. I. Mayo, K. O'Grady, P. E. Kelly, J. Cambridge, I. L. Sanders, T. Yogi, and R. W. Chantrell, J. Appl. Phys. 69, 4733 (1991).

${ }^{28}$ M. El-Hilo, K. O'Grady, P. I. Mayo, I. L. Sanders, and J. K. Howard, IEEE Trans. Magn. 28, 3283 (1992).

${ }^{29}$ M. El-Hilo, K. O’Grady, R. W. Chantrell, I. L. Sanders, M. M. Yang, and J. K. Howard, IEEE Trans. Magn. 27, 5061 (1991).

${ }^{30}$ R. A. McCurrie, Ferromagnetic Materials Structure and Properties (Academic, San Diego, CA, 1994). 